\title{
Modeling and Designing Self-Organized Aggregation in a Swarm of Miniature Robots
}

\author{
Nikolaus Correll ${ }^{1}$ and Alcherio Martinoli ${ }^{2 *}$ \\ ${ }^{1}$ Department of Computer Science \\ University of Colorado at Boulder, Boulder, CO, USA \\ 2 Distributed Intelligent Systems and Algorithms Laboratory \\ École Polytechnique Fédérale Lausanne, Switzerland.
}

February 14, 2011

\begin{abstract}
We model the dynamics of self-organized robot aggregation inspired by a study on the aggregation of gregarious arthropods. In swarms of German cockroaches, aggregation into clusters emerges solely from local interactions between the individuals, whereas the probabilities to join or leave a cluster are a function of the cluster size. We propose a non-spatial population dynamics model that keeps track of the number of robots in clusters of specific size using control parameters of the individual robots and the probability of detecting another robot in the environment. The model is able to quantitatively and qualitatively predict the dynamics observed in extensive realistic multi-robot simulation, and provides qualitative agreement with data obtained from aggregation of Blattela germanica larvae. In particular, we show by analysis, numerical and realistic simulation that the emergence of a single aggregate requires a minimal communication range between individuals.
\end{abstract}

\section{Introduction}

Aggregation processes are ubiquitous in a multitude of domains ranging from physics (Zangwill 2001) and biology (Parrish \& Hammer 1997), to swarm robotic

* Parts of the results presented in this paper have been shown in N. Correll and A. Martinoli. Modeling Self-Organized Aggregation in a Swarm of Miniature Robots. In IEEE 2007 International Conference on Robotics and Automation Workshop on Collective Behaviors inspired by Biological and Biochemical Systems, Rome, Italy, 2007. Both authors were sponsored by a Swiss NSF grant (contract Nr. PP002-68647). 
systems (Martinoli, Ijspeert \& Mondada 1999). The processes responsible for aggregation are likely to share similarities on different scales, from proteins, to social insects, and mammals (Whitesides \& Grzybowski 2002), suggesting a common methodological framework for modeling their dynamics. In the case of "self-organized" aggregation (Camazine, Deneubourg, Franks, Sneyd, Theraulaz \& Bonabeau 2001), which is a result from purely local interactions without central control or global information exchange, and an intrinsic amount of randomness, analysis of the resulting complex dynamics becomes cumbersome. Formal modeling of the underlying processes might lead not only to a better understanding of natural processes, e.g., the aggregation dynamics of gregarious insects (Jeanson, Rivault, Deneubourg, Blanco, Fournier, Jost \& Theraulaz 2005), but is also beneficial in an engineering context, for instance for designing nano-structures by self-assembly (Mermoud, Brugger \& Martinoli 2009), self-organized building processes (Martinoli et al. 1999), selforganized bacteria (Basu, Gerchman, Collins, Arnold \& Weiss 2005) or mixed animal-robot societies (Halloy, Amé, Detrain, Caprari, Asadpour, Correll, Martinoli, Mondada, Siegwart \& Deneubourg 2007) as well as determining their parameters (Correll \& Martinoli 2006). Also, aggregation can be understood as an important collective behavior in swarm robotics, as it might be the prerequisite for more complex collective tasks that rely on local interactions.

In self-organized aggregation phenomena, structures emerge out of continuous aggregation and disaggregation of clusters. In order for sophisticated structures to emerge, the behavior of the participating agents needs to change as a function of the aggregate. This can be achieved implicitly or explicitly. In chemical self-assembly building blocks within cyclic aggregates have a smaller probability to leave the aggregate as this involves the break of two chemical bonds at the same time vs. only one in non-cyclic aggregates (Mermoud et al. 2009). Social insects instead explicitly modulate their behavior based on their perception of the environment (Camazine et al. 2001). Examples involving robot swarms include aggregation of pucks (Martinoli et al. 1999, Agassounon, Martinoli \& Easton 2004), and collective decisions in mixed animal-robot societies (Halloy et al. 2007). While preferential attachment in aggregation of pucks is implicit by the increased detection area of larger clusters, in collective decisions - in (Halloy et al. 2007) robots and cockroaches collectively decide between two different shelters - both insects and robots join or leave an aggregate as a function of the estimated cluster size.

\subsection{Contribution of this paper}

We develop a probabilistic macroscopic model based on (Agassounon \& Martinoli 2002) and (Wang \& Wu 2005) for modeling the dynamics of a self-organized aggregation process that has been observed in gregarious arthropods (Jeanson et al. 2005) and has been successfully implemented in miniature robots in the past (Garnier, Jost, Jeanson, Gautrais, Grimal, Asadpour, Caprari \& Theraulaz 2008). In order to study the effect of various design parameters, including the behavioral parameters of the robot and its communication range, on the ag- 
gregation performance, we perform analysis of the macroscopic equations, numerical simulations, and realistic simulations. We show that solely tuning the behavioral parameters to that of the cockroach is not sufficient for aggregation into a single cluster, but robots need a minimum combination of communication range and locomotion speed in order to aggregate into a single cluster when using probabilistic aggregation rules. By this, we show that the proposed model can be used as a design tool for deriving the minimal required speed and/or communication range in order to achieve a specific average cluster size distribution. We also show that the proposed model can potentially be used to validate hypothesis on the average speed and communication range of the model natural system by comparing model prediction with data from cockroach aggregation experiments qualitatively.

\subsection{Related work: models for aggregation}

Our probabilistic modeling approach is complementary to deterministic models for aggregation from a systems and control perspective (Jadbabaie, Lin \& Morse 2003, Tanner, Jadbabaie \& Pappas 2005) as well as models for selfassembly using graph grammars (Klavins 2007). In the aggregation and flocking literature (flocking is conceptually similar to aggregation but involves also a coordinated motion of the aggregate), the emerging graph structure that represents the local neighborhood relations between agents as well as the agent dynamics, are explicitly modeled. This in turn allows to prove properties such as convergence analytically by assuming perfect sensors and actuators including range and bearing on the robotic platform. In our models, the graph structure is only implicitly modeled by the degree of each aggregate. In the grammarbased self-assembly literature, grammars determine the possible ways agents can inter-connect. The population dynamics of the resulting aggregates can then be described using non-spatial reaction-diffusion equations, whose coefficients are determined from spatial simulation or experimental data (Klavins 2007). In our approach, reaction coefficients are explicitly derived from the probabilistic robot controller, sensor and actuator noise, and the geometric properties of the environment. Also, instead of modeling particular instances of the system, our approach predicts the likelihood for the system to be in a particular state by tracking the average number of robots in clusters of different sizes. The model proposed in this paper is based on our previous work on aggregation of pucks by swarms of miniature robot (Martinoli et al. 1999), for which we derived an analytical model in (Agassounon \& Martinoli 2002, Agassounon et al. 2004), and which have also been studied in (Kazadi, Abdul-Khaliq \& Goodman 2002). The algorithms presented in these papers are similar to those presented here as clusters are aggregated and segregated probabilistically by mobile robots, although the pucks are not mobile themselves.

Another perspective on aggregation stems from work on maintaining wireless coverage using minimalist robots. For instance, Poduri \& Sukhatme (2007) propose an algorithm and analysis for coalescence of a team of robots to a static gateway based on random walk. As robots never resume motion once they 
stopped close to the gateway, this work is an instance of aggregation without edge deletion. An algorithm, in which clusters not only grow but might also shrink is presented in (Correll, Rus, Bachrach \& Vickery 2009). In this work we also use a probabilistic model for expressing the likelihood that all robots are part of a single aggregate as a function of robot control parameters. Similarly, algorithms for maintaining connectivity in a robot swarm described in (Nembrini, Winfield \& Melhuish 2002) have been modeled using a probabilistic model similar to that presented in this paper by Winfield, Liu, Nembrini \& Martinoli (2008).

Aggregation dynamics of systems in which aggregates can not only grow but also disperse have been proposed by (Agassounon \& Martinoli 2002, Kazadi et al. 2002, Lerman \& Shehory 2000, Wang, Liu \& Jin 2003, Matthey, Berman \& Kumar 2009) and have applications beyond the swarm-robotic domain such as coalition formation in large-scale electronic markets (Lerman \& Shehory 2000), load-balancing in grid computers (Wang et al. 2003), and molecular selfassembly (Wang \& Wu 2005). Aggregation processes with reversible population dynamics are also of interest to model chemical reaction networks, which are usually described by continuous-time differential equations (Feinberg 1995). For instance, Adleman, Gopalkrishnan, Huang, Moisset \& Reishus (2008) prove uniqueness and global convergence of equilibria in reversible, atomic systems. Similarly, Anderson \& Shiu (2010) prove global convergence of instances of weakly-reversible systems under certain conditions.

\section{Aggregation in Gregarious Arthropods}

The aggregation of larvae of the German cockroach (Blattella Germanica) serves as the behavioral model for robot aggregation in this paper. Cockroach behavior is known to be gregarious, i.e., individuals tend to associate with others of their kind, and aggregation is known to be mediated by only local interactions (Jeanson et al. 2005) between individuals.

Aggregation of a swarm of cockroaches in a bounded arena emerges as follows. Cockroaches move randomly through the arena (Jeanson, Blanco, Fournier, Deneubourg, Fourcassié \& Theraulaz 2003), eventually stop, and aggregate into clusters of different sizes, in which every cockroach can sense the presence of at least one other cockroach. Clusters are not persistent, because cockroaches might resume movement and quit the cluster.

The behavioral parameters as a function of cluster size have been measured for Blattella Germanica larvae by Jeanson et al. (2005). In their experiments, Jeanson et al. used first-instar larvae (24h old) in a circular arena and examined interaction in groups of two to four larvae, and trajectories of individual cockroaches. They show that the average time to rest within a cluster is a function of its size, where larger clusters are preferred over smaller ones.

Assuming that mutual perception takes place solely using their antennae, the rate at which a larva would stop when it perceived 1, 2 and 3 cockroaches within its perception radius has been measured in a suite of experiments. The rate 
Table 1: Probabilities to stop $\left(p_{n}^{\text {join }}\right)$ and restart $\left(p_{n}^{\text {leave }}\right)$ searching during one time step $(T=1 s)$ as a function of the number of neighbors within perception range.

\begin{tabular}{lll}
\hline$n$ & $p_{n}^{\text {join }}$ & $p_{c}^{\text {leave }}$ \\
\hline 0 & 0.03 & n.a. \\
1 & 0.42 & $1 / 49$ \\
2 & 0.5 & $1 / 424$ \\
3 & 0.51 & $1 / 700$ \\
$4+$ & 0.51 & $1 / 1306$ \\
\hline
\end{tabular}

of stopping when no other larvae are nearby was estimated using experiments involving a single individual. Similarly, the time until an individual left a cluster of $1,2,3$ and 4 cockroaches was used to calculate the rate at which cockroaches spontaneously left a cluster. Here, Jeanson showed that the resting times follow a bimodal distribution that correspond to short and long resting periods.

In this paper, we are using the behavioral data collected from the cockroach to achieve aggregation in a swarm of miniature robots. For simplicity, we chose a unimodal distribution by averaging the resting times provided in (Jeanson et al. 2005). Also, although cockroaches in (Jeanson et al. 2005) show varying behavior as a function of their location within the arena (close to the center or close to the walls), these effects are neither implemented nor modeled in this paper.

\section{Robot Behavior}

Following the behavioral model described in (Jeanson et al. 2005), every robot is either moving or resting. For a finite number of robots $N_{0}$ in the arena, a robot can be part of a cluster of size $1,2,3, \ldots, N_{0}$. Both the transition probability for entering the rest state from the move state $\left(p^{\text {join }}\right)$ and resuming to move $\left(p^{\text {leave }}\right)$ are given by a non-linear function that is a function of the robots nearby (see Table 1). Probabilities have been adopted from behavioral parameters of the insects. However, while the values of $p^{\text {join }}(j)$ are identical to those reported in (Jeanson et al. 2005), $p^{\text {leave }}(j)$ is the average leaving probability for short and long stops that lead to a double-exponential distribution in (Jeanson et al. 2005). Note that the probability $p^{\text {join }}(0)$ is the probability to stop when no neighbors are present.

When moving, the robot senses a part of the arena and eventually encounters other robots with which it might aggregate. The area which the robot sweeps at every time interval is given by its communication range that is approximated by a disc with a certain radius in this paper.

The swarm (the ensemble of robots) aggregates within the environment in clusters of different size. Due to the robots' preference to stay with larger 
clusters (given by the behavioral parameters extracted from cockroach experiments), robots eventually aggregate into a single aggregate. This behavior is independent of the initial distribution of robots in the environment.

Notice that as $p^{\text {leave }}(j)>0$ the single cluster is not a stable state, but robots continue to steadily leave and re-joining the cluster.

\section{A Probabilistic Model for Aggregation Dy- namics}

The individual behavior described above can be represented by a Markov dynamical system for every individual, whereas the collective behavior can be described by averaging over the states of all individuals.

\subsection{Individual Dynamics}

We describe the dynamics of each individual $v$ by a Markov chain with a set of states $\mathcal{X}$.

The state space $\mathcal{X}^{(v)}$ is discrete, finite, and reflects the size of the aggregate that a robot is part of. A static robot that does not have any neighbors (other robots in communication range) is considered an aggregate of size one. For a finite number $N_{0}$ of robots, the state space is thus given by

$$
\mathcal{X}^{(v)}=j \in\left\{0,1, \ldots, N_{0}\right\},
$$

with $j$ denoting the size of the aggregate the robot is part of, and $\mathcal{X}^{(v)}=0$ denoting a searching robot.

The conditional probability for a moving robot to join an aggregate of size $j$ when searching is given by

$$
P\left(\mathcal{X}^{(v)}=j \mid \mathcal{X}^{(v)}=0\right): \mathbb{Z}_{+} \rightarrow[0,1],
$$

and will be denoted by $p^{\text {join }}(j)$ in the remainder of this paper. Similarly the conditional probability for a robot to leave an aggregate of size $j$ and resume search is given by

$$
P\left(\mathcal{X}^{(v)}=0 \mid \mathcal{X}^{(v)}=j\right): \mathbb{Z}_{+} \rightarrow[0,1]
$$

and will be denoted by $p^{\text {leave }}(j)$. As the formalism above assumes that all robots in an aggregate can sense the number of robots in this aggregate, we also refer to an aggregate as a clique.

Notice that a robot might change its state not only due to its own action, but also when another robot is added or removed from the aggregate it is part of.

The state space and possible state transitions are summarized graphically in Figure 1. This state-machine is similar to that presented in (Martinoli et al. 1999) for the dynamics of clusters of seeds, but is extended by an additional state that describes a searching robot. 


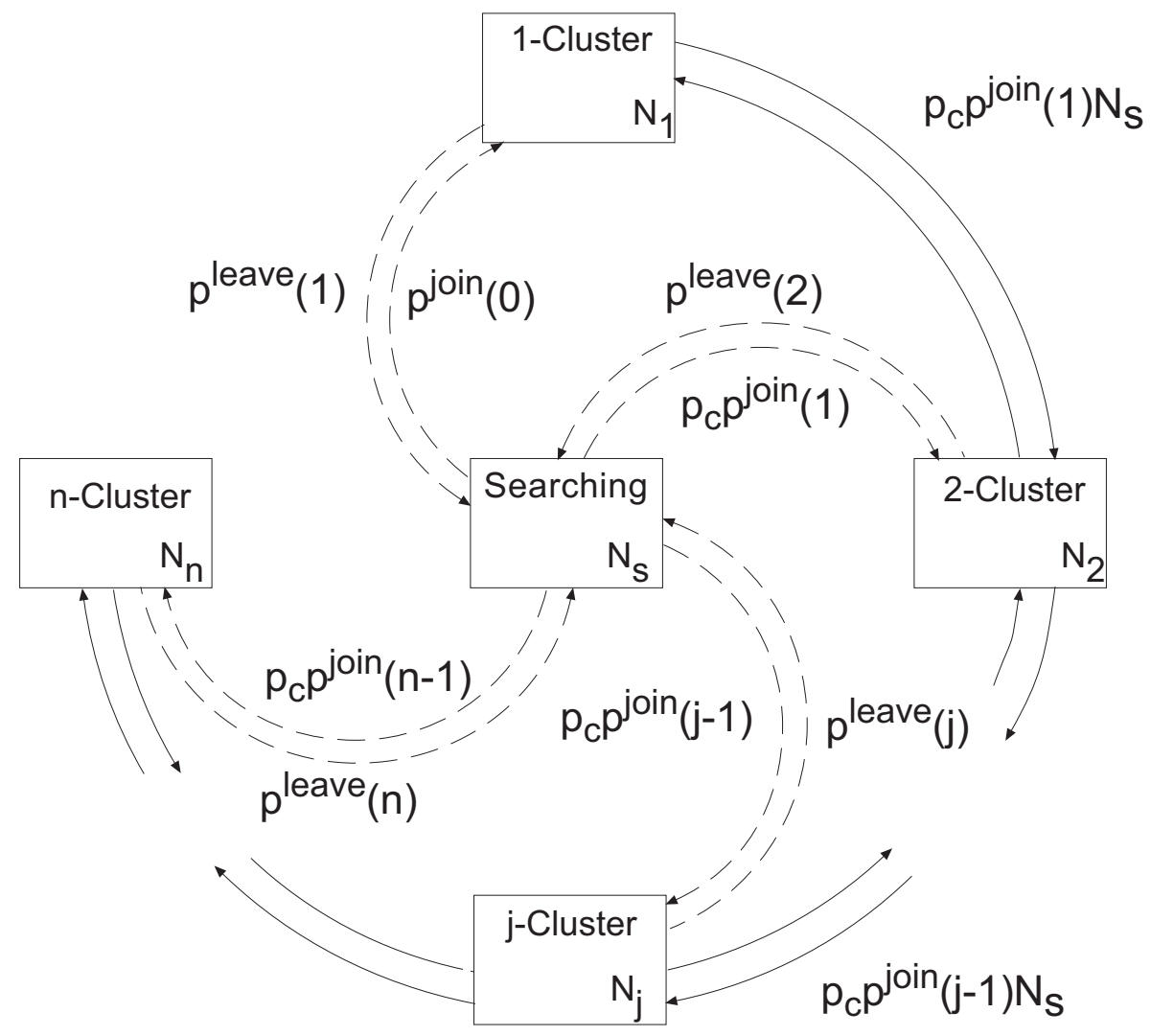

Figure 1: State transition diagram of an individual robot. 


\subsection{Mobility}

Assuming an uniform distribution of objects in the arena, constant speed, and constant sensing range, we can approximate the detection of a robot within the arena by an encountering probability (see for instance (Agassounon et al. 2004, Martinoli, Easton \& Agassounon 2004, Correll \& Martinoli 2006), and references therein).

Definition: Encountering Probability An individual moving with constant speed and constant sensor range within a bounded arena, has a constant probability $p_{c}$ to encounter another individual of constant size and appearance at every time step of length $T$. In environments populated with $N$ individuals that are uniformly distributed, the probability to encounter one of them is calculated by $N p_{c}$ (linear superposition of encountering probabilities).

In order for this definition to be valid, the following assumptions have to hold (Martinoli et al. 2004, Agassounon et al. 2004):

- The distribution of robots and objects in the environment is uniform, i.e., the probability for a robot to encounter another robot or an object is constant through-out the environment.

- The density of robots and objects in the environment is so low that detection areas of robots and objects do not overlap, and the probability to encounter a robot or object is proportional to its area.

Notice that correlated random walk (Jeanson et al. 2003) in a bounded arena eventually leads to an uniform probability density function for the location of every cockroach in the environment (Berg 1983). Also, in our experiments we did not implement the wall-following behavior that is observed for real cockroaches (Jeanson et al. 2005), and which promotes aggregation close to the arena border.

In (Martinoli et al. 2004, Correll \& Martinoli 2004) we showed that the following relation holds for the probability $p_{c}$ :

$$
p_{c} \sim \frac{1}{A_{\text {total }}} v_{r} w_{d} T,
$$

with $A_{\text {total }}$ the area of the arena, $v_{r}$ the average speed of an individual, $w_{d}$ the individual's detection width, i.e., the width it sweeps with its sensors while moving (the sensor range is equivalent to the robot's communication range in this case), and the time discretization of the system $T$. Thus, $p_{c}$ is proportional to an individual's speed (given by $v_{r}$ ) as well as its communication range (given by $w_{d}$ ).

We note that in the aggregation system studied in this paper, the agent speed, and therefore $p_{c}$, are analogous to the temperature in a chemical reaction, where higher temperatures correspond to higher molecule mobility. 


\subsection{Collective Dynamics}

The behavior of the individual robot can be described by a Markov chain that describes the probability for a robot to be in a specific state, i.e., the likelihood for a robot to be in a specific state can be described by a random variable. For the probability of a random variable $p$ to have the value $j$ at time interval $k$, one can write the following time-discrete Master equation

$$
p_{j}(k T+T)=p_{j}(k)+\sum_{j^{\prime} \in \mathcal{X} \backslash j}\left(p_{j^{\prime} j}(k T+T) p_{j^{\prime}}(k)-p_{j j^{\prime}}(k T) p_{j}(k T)\right)
$$

where $p_{j^{\prime} j}(k T+T)$ is the conditional probability that the system will be in state $j$ at time $k T+T$ when in state $j^{\prime}$ at time $k T$. Notice, that $p_{j^{\prime} j}(k T+T)$ can be also understood as the transition probability of state $j$ to $j^{\prime}$ of a Markov chain with states $\mathcal{X} . T$ is the time discretization of the system and $k$ is indexing the time-steps. For brevity, $T$ is omitted in the remainder of this paper and we write $p_{j}(k)$ instead of $p_{j}(k T)$.

Using $p_{j}(k)$ and the total number of individuals $N_{0}, p_{j}(k)$ can also be interpreted as the fraction of robots in state $j$ and $N_{0} p_{j}(k)$ yields the the expected number of robots in state $j$. For brevity, it is written

$$
N_{j}(k)=N_{0} p_{j}(k)
$$

Using this notation, 5 can be written as

$$
N_{j}(k+1)=N_{j}(k)+\sum_{i}\left(p_{i j}(k+1) N_{i}(k)-p_{j i}(k) N_{j}(k)\right)
$$

which is a discrete-time Rate equation. This derivation is explained in more detail in (Lerman, Martinoli \& Galystan 2005).

We will now develop a set of difference equations that summarize the average behavior of an ensemble of Markov chains, and thus keep track of the number of aggregates of size 1 to $N_{0}$. This equation set is similar to that we proposed in (Agassounon \& Martinoli 2002) except that elements in the aggregate can also be mobile, which leads to an additional state. An equivalent system is described in (Wang \& Wu 2005), which additionally considers a continuous addition of elements, motivated by a molecular beam epitaxy scenario in which new molecules are continuously provided by a beam.

The ensemble of individuals as well as its structural properties is now represented by a set of difference equations, which keeps track of the average number of individuals in each state. Inflow and outflow of each state represents the average fluctuations between states and are given by the probability for a state transition to occur and the number of robots in other states.

Relying on Definition 1 and its assumptions, the average number of robots $N_{j}(k+1)$ in an aggregate of size $j$ (with $1<j<N_{0}$ ) at time $k+1$, is then 
given by the following difference equation

$$
\begin{aligned}
N_{j}(k+1)= & N_{j}(k)+p_{c} N_{j-1}(k) N_{s}(k) p^{\text {join }}(j-1) j \\
& +p^{\text {leave }}(j+1) N_{j+1}(k) j \\
& -p_{c} N_{j}(k) N_{s}(k) p^{\text {join }}(j) j \\
& -p^{\text {leave }}(j) N_{j}(k) j
\end{aligned}
$$

The term $p_{c} N_{j-1}(k) N_{s}(k) p^{j o i n}(j-1)$ corresponds to the number of searching robots $N_{s}(k)$ that encounter one of the robots in a cluster of size $j-1$ (probability $p_{c} N_{j-1}(k)$ ), and decide to join this robot's cluster with probability $p^{\text {join }}(j-1)$. Then, $j$ more robots would be in a cluster of size $j$. This is also the case when any robot in a cluster of size $j+1$ leaves its cluster with probability $p^{\text {leave }}(j+1)$.

The number of robots in a cluster of $j$ diminishes by $j$ when a searching robot joins a cluster of size $j$ (with probability $p_{c} N_{j}(k) N_{s}(k) p^{j o i n}(j)$ ), or when a robot leaves a cluster of $j$ with probability $p^{\text {leave }}(j)$.

Notice that the ensemble of $N_{j}(k), j=\left\{0, \ldots, N_{0}\right\}$ corresponds to the cluster size distribution of the clusters in the environment.

For an aggregate consisting of $N_{0}$ robots, (8) simplifies to

$$
\begin{aligned}
N_{N_{0}}(k+1)= & N_{N_{0}}(k) \\
& +p^{\text {join }}\left(N_{0}-1\right) N_{N_{0}-1}(k) p_{c} N_{s}(k) N_{0} \\
& -p^{\text {leave }}\left(N_{0}\right) N_{N_{0}}(k) N_{0}
\end{aligned}
$$

as there exist no aggregates of size $N_{0}+1$, nor any moving robots in this case. The number of aggregates of size one, at time $k+1$ is given by

$$
\begin{aligned}
N_{1}(k+1)= & N_{1}(k) \\
& -p^{\text {leave }}(1) N_{1}(k) \\
& +p^{\text {leave }}(2) N_{2}(k) \\
& +p^{\text {join }}(0) N_{s}(k) \\
& -p^{\text {join }}(1) N_{1}(k) p_{c} N_{s}(k)
\end{aligned}
$$

For a constant number of robots, we can calculate the number of moving robots by

$$
N_{s}(k+1)=N_{0}-\sum_{n=1}^{N_{0}} N_{n}(k+1)
$$

We note that maintaining a dedicated equation for every possible cluster-size seems to let the resulting analysis scale poorly. Due to the repetitive pattern of the equation system, numerical solutions are easily to obtain and allow to get quantitative insight into the state-space dynamics orders of magnitudes faster than using agent-based simulation for the same number of agents (see below), however. 


\subsubsection{Steady-State Analysis}

The equation system (8)-(10) can be cast into the system described in (Wang $\& \mathrm{Wu} 2005$ ). In (Wang \& Wu 2005), it is shown that all solutions of (8)-(10) are always positive for positive values of $p^{\text {join }}(j), p^{\text {leave }}(j)$ and $p_{c}$. They also show that its equilibria are unique and stable when either $p^{\text {leave }}(j)=0$, i.e., no robot ever leaves a cluster, or $p^{j o i n}(j)=0$, i.e., no robot ever joins a cluster.

In this paper, we are interested in how different choices of $p^{\text {join }}(j), p^{\text {leave }}(j)$ and $p_{c}$ affect the behavior of the system at steady-state. Here, our goal is to derive conditions for $p^{\text {join }}(j), p^{\text {leave }}(j)$ and $p_{c}$ that help us design systems that aggregate into as large aggregates as possible. Although deriving analytical, closed-form equations for the steady-state of the difference equations is very difficult - if not infeasible - due to the high degree of nonlinearity of the resulting system of equations (except for trivial numbers of robots), we show by inspection of the system dynamics when $N_{j}^{*}(k+1)-N_{j}^{*}=0$ that

1. There exist a unique steady-state solution for $N_{j}^{*}$ for every $N_{s}^{*} \in\left[0, N_{0}\right]$

2. The proportion of robots in clusters at different size is bound by the ratio of consecutive elements of $p^{\text {join }}(j)$ and $p^{\text {leave }}(j)$

3. The system behavior can change between dispersion and aggregation simply by tuning of $p_{c}$

Theorem 4.1 For the system with the dynamics defined in (8), (9) and (10), there exist a unique steady-state solution for all $N_{j}^{*}$ for every $N_{s}^{*} \in\left[0, N_{0}\right]$

Proof Let $N_{j}^{*}$ be the expected number of robots in a cluster of size $j$ at steadystate. By solving (8) for $N_{j}(k+1)-N_{j}(k)=0$, we obtain

$$
N_{j-1}^{*} p_{c} N_{s}^{*} p^{j o i n}(j-1)-N_{j}^{*}\left(p_{c} N_{s}^{*} p^{j o i n}(j)+p^{\text {leave }}(j)\right)+N_{j+1}^{*} p^{\text {leave }}(j+1)=0
$$

With $j=\left[1, \ldots, N_{0}\right]$ and $N_{s}^{*}$ as a constant this a linear equation system with $N_{0}+1$ unknowns, but provides only $N_{0}$ equations. Thus, solutions for $N_{j}^{*}$ can be obtained by solving a linear equation system in which we fix one value, e.g., $N_{s}^{*}=\left[0, \ldots, N_{0}\right]$, and calculate the corresponding steady-state.

As solving the system for all possible combined values of $p^{\text {join }}(j)$ and $p^{\text {leave }}(j)$ is infeasible, we will investigate the system by considering joining and leaving actions independently from each other. We also notice that the existence of an equilibrium for a certain $N_{s}^{*}$ does not allow to draw conclusions on its stability. For instance $N_{s}^{*}=N_{0}$ is a possible state for the system for an infinite number of different $p^{\text {join }}(j)$ and $p^{\text {leave }}(j)$, but is only a stable equilibrium for the subset where $p^{\text {join }}(j)=0$. However, one can show that a unique, stable equilibrium exist by applying Feinberg's Zero Deficiency theorem (Feinberg 1995), which has been shown in (Matthey et al. 2009) for an equivalent system. For a numerical stability analysis, the reader is referred to (Wang \& Wu 2005), who derive stability analytically for $N_{0} \leq 3$ and numerically for $N_{0}>3$. 
Lemma 4.2 For the system with the dynamics defined in (8), (9) and (10) with $p^{\text {join }}(j)>0$, but $p^{\text {leave }}(j)=0, N_{j}^{*}$ is not a function of $N_{s}^{*}$.

Proof We denote steady-states of the system with $p^{\text {leave }}(j)=0$ by $N_{j}{ }^{*}$. When considering (12) for $p^{\text {leave }}(j)=0$, i.e., no robots ever leave, we obtain

$$
\hat{N}_{j}^{*}=\frac{p^{j o i n}(j-1)}{p^{j o i n}(j)} N_{j-1}^{\prime}
$$

and

$$
{N_{1}}^{*}=\frac{p^{\text {join }}(0)}{p^{\text {join }}(1) p_{c}}
$$

using (10) for $p^{\text {leave }}(j)=0$. By plugging in (14) into (13) and calculating $N_{2}^{*}$, and hence $N_{3}^{*}$ and so forth, the solution for $N_{j}^{*}$ is given by

$$
N_{j}^{*}=\frac{p^{\text {join }}(0)}{p^{\text {join }}(j) p_{c}}
$$

Thus, the ratio of robots per cluster-size is solely determined by $p^{\text {join }}(0), p^{\text {join }}(j)$ and $p_{c}$.

Remark Looking at (9) at steady-state for $p^{\text {leave }}(j)=0$, we obtain

$$
p^{j o i n}\left(N_{0}-1\right) N_{N_{0}-1}^{*^{\prime}} p_{c} N_{s}^{*} N_{0}=0
$$

As $p^{\text {join }}(j)>0, p_{c}>0, N_{0}>0$ by definition, it follows that ${N_{N_{0}-1}^{\prime}}^{*}>0$ using (15). Therefore, $N_{s}^{*}$ is equal to zero at steady-state.

Remark All $\hat{N}_{j}^{*}=\frac{p^{j o i n}(0)}{p^{j o i n}(j) p_{c}}$ are stable equilibria of the system as clusters cannot disperse with $p^{\text {leave }}(j)=0$, see also (Wang \& Wu 2005).

Lemma 4.3 For the system with the dynamics defined in (8), (9) and (10) with $p^{\text {leave }}(j)>0$, but $p^{j o i n}(j)=0, N_{j}^{*}$ is not a function of $N_{s}^{*}$.

Proof We denote steady-states of the system with $p^{j o i n}(j)=0$ by $\grave{N}_{j}{ }^{*}$. By solving for the special case of $N_{N_{0}}^{*}$ and $p^{\text {join }}(j)=0$, we obtain

$$
0=p^{\text {leave }}\left(N_{0}\right) N_{N_{0}}^{*} N_{0}
$$

where all terms except $N_{N_{0}}^{*}$ are positive by definition. By using the recurrence equation

$$
N_{j+1}^{\grave{*}}=\frac{p^{\text {leave }}(j)}{p^{\text {leave }}(j+1)} \grave{N}_{j}^{*}
$$

which can be derived in a similar fashion as for the case with $p^{\text {leave }}(j)=0$, we can see that actually all $\hat{N}_{j}^{*}$ are zero at steady-state and therefore $\hat{N}_{s}^{*}=N_{0}$. 
Remark As the system will reach $\grave{N}_{s}^{*}=N_{0}$ independent of the values of $p^{\text {leave }}(j)$ and as there is no aggregation possible because of $p^{j o i n}(j)=0, \hat{N}_{s}^{*}=$ $N_{0}$ is a stable equilibria (see also (Wang \& Wu 2005)).

The system that combines preferential attachment and detachment therefore balances two different behaviors: aggregation into fixed clusters that are distributed according to the values of $p^{\text {join }}(j)$ and dispersion into a steady-state where all robots are searching $\left(\grave{N}_{s}^{*}=N_{0}\right)$. As detachment does not contribute to cluster growth, and as attachment and detachment can be considered independent behaviors, i.e., a robot either detaches or attaches, we conclude that the maximum cluster size at steady-state is bound by (15). We conjecture that detachment actually only helps to increase the mixing in the system by increasing $N_{s}^{*}$, i.e., the number of robots that are searching. Here, higher probabilities for leaving a smaller cluster over a larger cluster might bias the system to promote larger clusters. There is a trade-off, however, with the fact that too high probabilities to leave a cluster will not give this cluster enough time to grow. We will address this issue further down.

Theorem 4.4 For the system with the dynamics defined in (8), (9) and (10), the proportion of robots in clusters at different size is bound by the ratio of consecutive elements of $p^{\text {join }}(j)$ and $p^{\text {leave }}(j)$.

Proof By inspection of (12) we observe that all terms are larger than zero by definition. We can therefore establish the following relationship between cluster sizes at steady-state and joining and leaving probabilities: if $N_{j-1}^{*} p^{j o i n}(j-$ 1) $<N_{j} p^{\text {join }}(j)$, i.e., if smaller clusters grow slower than larger clusters, then it follows $N_{j+1}^{*} p^{\text {leave }}(j+1)>N_{j}^{*} p^{\text {leave }}(j)$ in order for (12) to be zero. Likewise, if $N_{j-1}^{*} p^{j o i n}(j-1)>N_{j} p^{j o i n}(j)$, i.e., if smaller clusters grow faster than larger clusters, then it follows $N_{j+1}^{*} p^{\text {leave }}(j+1)<N_{j}^{*} p^{\text {leave }}(j)$.

This leads to the following inequalities that the resulting steady-state will obey if smaller clusters grow slower than larger ones

$$
\frac{N_{j-1}^{*}}{N_{j}^{*}}<\frac{p^{\text {join }}(j)}{p^{\text {join }}(j-1)} \quad \text { and } \quad \frac{N_{j-1}^{*}}{N_{j}^{*}}<\frac{p^{\text {leave }}(j)}{p^{\text {leave }}(j-1)}
$$

and to

$$
\frac{N_{j-1}^{*}}{N_{j}^{*}}>\frac{p^{\text {join }}(j)}{p^{j \text { oin }}(j-1)} \quad \text { and } \quad \frac{N_{j-1}^{*}}{N_{j}^{*}}>\frac{p^{\text {leave }}(j)}{p^{\text {leave }}(j-1)}
$$

if smaller clusters grow faster than larger ones.

Based on the preceding theorems and lemmas, we will now show that

Theorem 4.5 For the system with the dynamics defined in (8), (9) and (10), the system behavior can change between dispersion and aggregation simply by tuning of $p_{c}$ as long as the inequalities for $p^{\text {join }}(j)$ and $p^{\text {leave }}(j)$ are satisfied. 

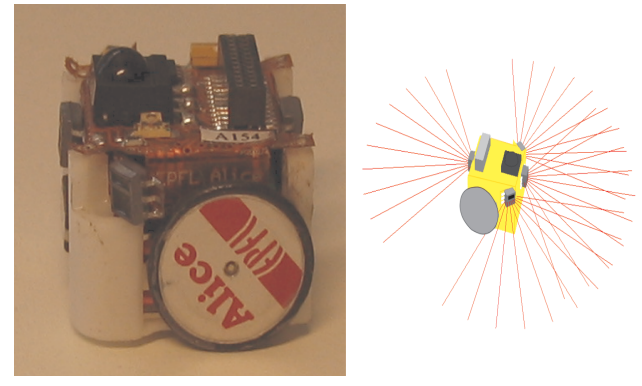

Figure 2: The miniature robot Alice (left), and its simulated counterpart (right).

Proof Solving (9) for $N_{N_{0}}(k+1)-N_{N_{0}}(k)=0$, we obtain

$$
\frac{N_{N_{0}}^{*}}{N_{N_{0}-1}^{*}}=\frac{p^{\text {join }}\left(N_{0}-1\right)}{p^{\text {leave }}\left(N_{0}\right)} p_{c} N_{s}^{*}
$$

We have shown in Lemmas 4.2 and 4.3 that $N_{s}^{*}$ is only a function of the values of $p^{\text {join }}(j)$ and $p^{\text {leave }}(j)$ and will assume a value in the interval of $\left[0, N_{0}\right]$. Thus, whether the system's behavior is governed by $N_{N_{0}}^{*}>N_{N_{0}-1}$, or $N_{N_{0}}^{*}<N_{N_{0}-1}$, which corresponds to aggregation into a super-cluster or aggregation into any other steady-state cluster distribution, respectively, can be controlled by $p_{c}$, provided that condition (19) from Theorem 2 is satisfied.

\section{Experimental Setup}

We wish to show that the macroscopic aggregation model developed above is indeed relevant to model the average dynamics of a physical system that aggregates based on probabilistic attachment and detachment, and moreover, that the results of the steady state analysis (Lemmas 4.2, 4.3 and Theorem 4.5) gives relevant guidelines for the design choices of such a system. As there a $N_{0}$ profiles $N_{j}(k)$ which describe the average number of robots in a cluster of size $j$, large amounts of experimental data are necessary for a quantitative comparison of model prediction and the behavior of a real system. We therefore rely on the realistic simulator Webots, which has shown to faithfully reproduce collective behavior of miniature robots in the past (Michel 2004, Martinoli et al. 2004) to gather data for simulated miniature robots. The behavior of the robots was here tuned based on behavioral data obtained from a systematical study with larvae of the species Blattela Germanica (Jeanson et al. 2005).

\subsection{Robotic Platform}

The Alice robot (Caprari \& Siegwart 2005) has a size of $2 \mathrm{~cm} \times 2 \mathrm{~cm} \times 2 \mathrm{~cm}$, a differential wheel drive that reaches speed of up to $4 \frac{\mathrm{cm}}{\mathrm{s}}$, four infrared distance sensors for obstacle detection (up to $3 \mathrm{~cm}$ ), and $4 \mathrm{Bit} / \mathrm{s}$ local communication up 


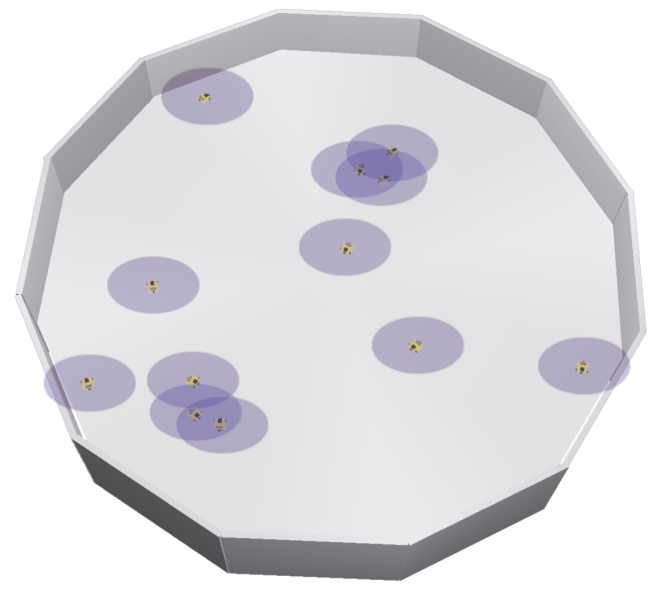

Figure 3: The communication range of each individual is shown by superimposed discs. The scenario shows six clusters of 1 individual, and two clusters of 3 individuals.

to $6 \mathrm{~cm}$, which can serve as well as crude low-range and bearing sensor (Figure 2 ). The robot as well as its sensors and actuators is faithfully implemented in Webots (see below).

In the simulator, robots have a speed of $4 \mathrm{~cm} / \mathrm{s}$ and can evaluate the number of neighbors using local communication (we assume that each robot has an unique ID or can randomly choose its ID from a sufficiently large set). Notice that behavioral probabilities in our model are independent from the robots' speed.

A random communication network topology is depicted in Figure 3 where the communication range (here $10 \mathrm{~cm}$ ) of each individual is depicted by a circular disc. We refer to the ensemble of robots that can communicate with themselves directly or indirectly as a cluster.

The arena has a diameter of $1 \mathrm{~m}$.

\subsection{Simulator}

The experimental setup and hardware described above was implemented in $W e$ bots (Michel 2004) a submicroscopic, realistic simulator that is able to accurately model the non-linear sensor and actuator characteristics of the Alice robot, including Gaussian noise on the sensors as well as wheel-slip.

For this case study, Webots simulations allow us to collect results about 3 to 4 times faster than in equivalent experiments involving real robots. Using a computational cluster equivalent to 35 Pentium IV processors, we are able to collect a sufficient amount of data for quantitatively studying the robots' performance and its distribution for different control parameters. Using this configuration, we obtained around 120 simulations per hour with up to 12 robots. 
In contrast, gathering quantitative data on the state-space dynamics using the macroscopic model takes only a few seconds for 12 agents, which corresponds roughly to four orders of magnitude speed-up when using the macroscopic model instead of the realistic simulator. In addition for being computationally more expensive due to faithful simulation of sensors and kinematics, a large number of experiments is needed in order to obtain smooth average data, as the number of possible states of the system is very large (all possible combinations of cluster sizes), and some conformations can be observed less often than others (e.g., there is more data available on clusters of size 1 than for clusters of size 12).

For all simulations, the robots were deployed with random position and orientation in the arena so that there communication radii do not intersect, i.e., all robots are initially searching $\left(N_{s}(0)=N_{0}\right)$.

\section{Results}

For all data presented in this paper, difference equations are solved by numerical integration for $3 \mathrm{~h}$ of simulated time (time discretization of the system $T=1 \mathrm{~s}$ ) with $N_{s}(0)=N_{0}=12$ as initial condition. For experimental reasons related to the Webots simulator we do not distinguish between moving robots and robots in clusters of size 1 . Therefore, we add up $N_{s}(k)$ and $N_{1}(k)$ when comparing simulation and modeling results.

First, we would like to test whether Theorem 4.5 holds. For this, we tested the steady-state behavior of our model for different values of $p_{c}\left(0 \leq p_{c} \leq 0.1\right)$, and plot the resulting cluster size distribution for selected values in Figure 4. We clearly observe a shift in system behavior from steady-states in which all robots are scattered to steady-states where robots tend to aggregate in a single cluster.

Second, we would like to test whether changing the communication range in the robotic simulator has the same effect. We performed 1500 runs in Webots for $3 \mathrm{~h}$ of simulated time each, and the number of robots in clusters of 1 to 12 individuals was counted every 10 seconds. The communication range, i.e., the maximal distance other agents can be sensed, was set to $7 \mathrm{~cm}, 10 \mathrm{~cm}$, and $12 \mathrm{~cm}$.

Indeed, results from simulation with communication ranges of $7 \mathrm{~cm}, 10 \mathrm{~cm}$ and $12 \mathrm{~cm}$ (Figure 5) show a similar trend. In addition, results from realistic simulation and numerical integration of the macroscopic model show quantitative agreement. Figure 6 compares results for a communication range of $10 \mathrm{~cm}$ in realistic simulation (Figure 5 ) and $p_{c}=0.0102$, which can be derived using (4), in the macroscopic model for the specific case of a cluster of 12 individuals. Specifically, we used the following values for calculating $p_{c}: v_{r}=0.04 \mathrm{~m} / \mathrm{s}$, $A_{\text {total }}=0.785 \mathrm{~m}^{2}$ and $w_{d}=0.2 \mathrm{~m}$, which corresponds to the programmed robot speed in the simulation, an arena of $1 \mathrm{~m}$ diameter, and a communication range of $10 \mathrm{~cm}$, respectively. Figure 6 also shows that the steady-state is only reached asymptotically.

We are also interested in understanding the relevance of the proposed model to cockroach aggregation. Figure 7, right, shows the temporal evolution of the 


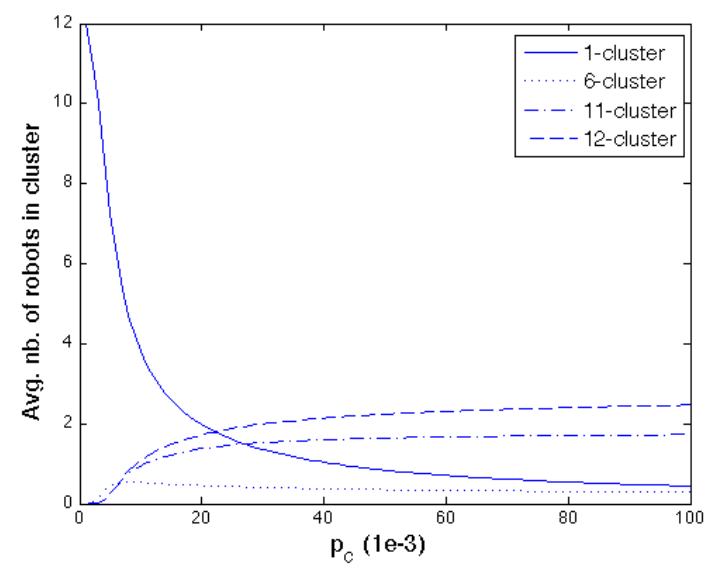

Figure 4: Cluster size at steady state as a function of the encountering probability $\left(0 \leq p_{c} \leq 0.1\right)$. Values are shown for the number of robots in a cluster of size 1 or searching, in a cluster of 6 , in a cluster of 11 , or in a cluster of 12 , the latter corresponding to all robots aggregating in a single aggregate. For a critical value of $p_{c}$, the system shows the tendency for aggregation to a single aggregate.

mean size of the largest aggregate in 20 experiments with 10 living cockroaches (from (Jeanson et al. 2005)). On the left of Figure 7, numerical solutions for the size of the average aggregate in a system of 10 elements are shown for different values of $p_{c}$ and the behavioral parameters from Table 1 . The encountering probability $p_{c}$ has been intentionally kept a free parameter of our system as we do not have experimental evidence of the communication range of the cockroach larvae in (Jeanson et al. 2005). Instead, (Jeanson et al. 2005) derives a possible detection distance of $w_{d, l}=12 \mathrm{~mm}$ based on measurements of the cockroaches' antenna length. Together with the mean speed of the larvae when outside of the periphery of the arena of $v_{l}=11 \mathrm{~mm} / \mathrm{s}$, and the arena diameter of $11 \mathrm{~cm}$ that has been used in Jeanson's experiment, we calculate an encountering probability $p_{c} \approx 0.017$. Indeed, the curve with $p_{c}=0.02$ in Figure 7 shows the closest qualitative and quantitative agreement with the experimental data.

Notice that the representation of the data in Figure 7 is different from those in the previous Figures, e.g., Figure 6 . Whereas our model describes the average number of robots in a cluster of size $j$, (Jeanson et al. 2005) provides measurements for the average of the largest cluster in their experiments. Although we cannot capture this quantity with our model, we believe that the average cluster size, i.e., $\sum_{j=0}^{N_{0}} j N_{j}(k)^{*} / N_{0}$ with $N_{0}(k)=N_{s}(k)$, is a reasonable approximation especially toward the end of the experiment, where the overall number of clusters becomes low. 


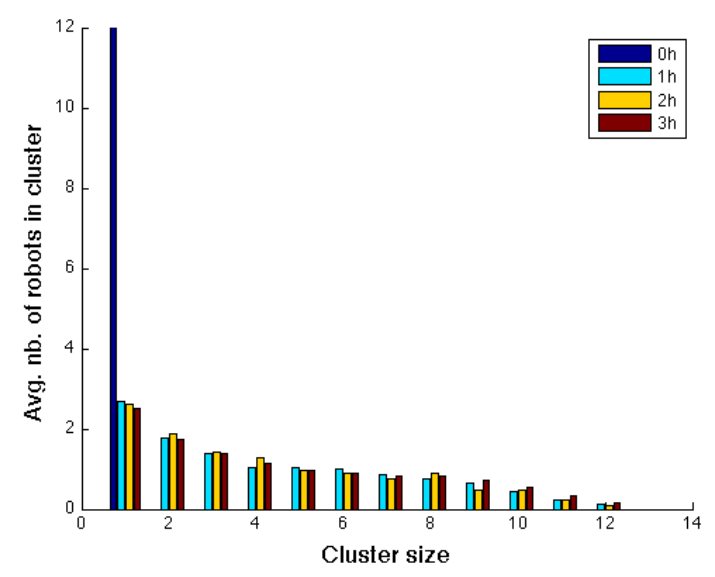

(a) $7 \mathrm{~cm}$ communication range

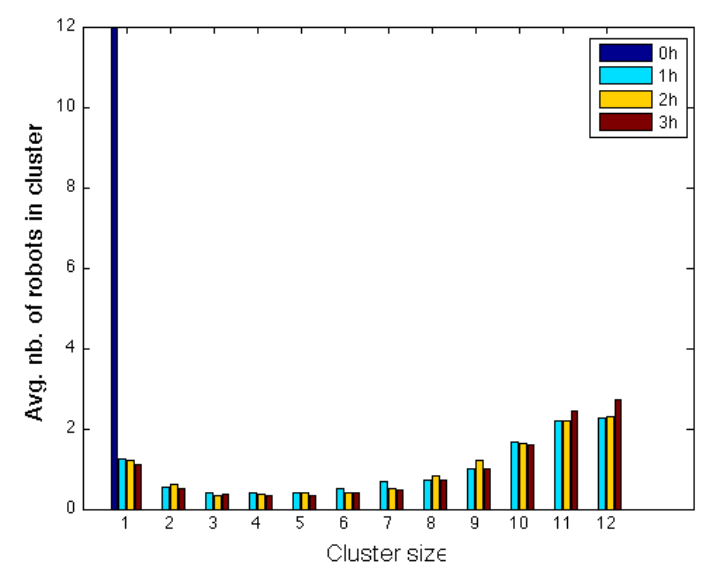

(b) $10 \mathrm{~cm}$ communication range

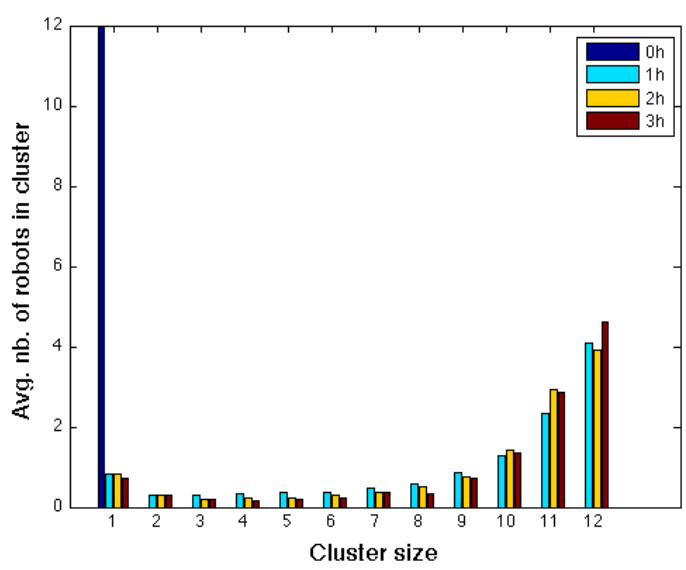

(c) $12 \mathrm{~cm}$ communication range

Figure 5: Average cluster size after $3 \mathrm{~h}$ of simulated time for $7 \mathrm{~cm}, 10 \mathrm{~cm}$ and $12 \mathrm{~cm}$ communication range (center to center). 1500 simulations per communication range, the cluster size was recorded every 10 seconds. 


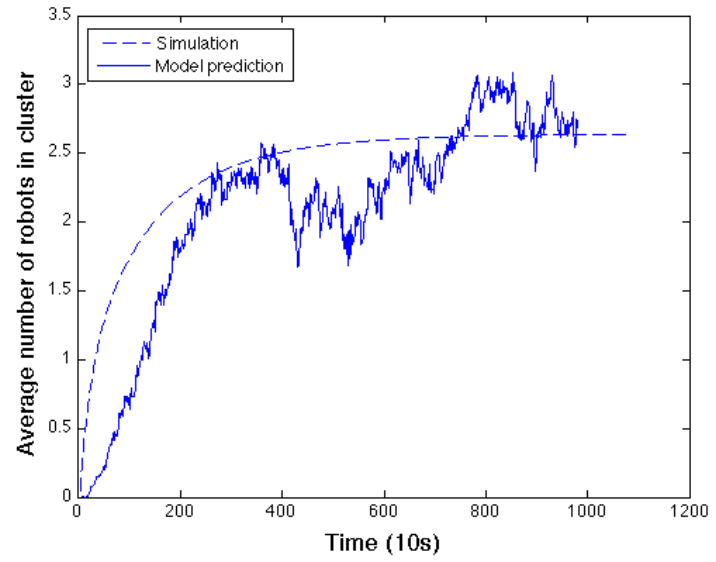

Figure 6: Model prediction using $p_{c}=0.0102$ given by (4) vs. simulation results (average over 1500 simulation) for the average number of robots in a cluster of 12 for $10 \mathrm{~cm}$ communication range (see Figure 5).
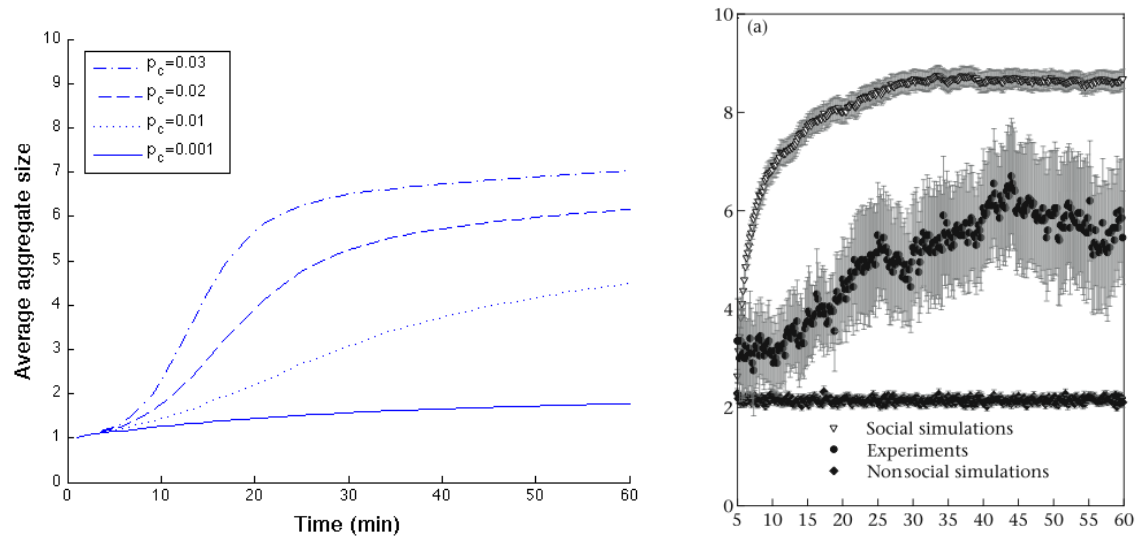

Figure 7: Left: Macroscopic simulation of a system of 10 agents with $p^{\text {join }}(j)$ and $p^{\text {leave }}(j)$ according to Table 1 showing the temporal evolution of the average cluster size for various $p_{c}$. Right: Experimental and simulation data from cockroach aggregation. Reprinted from (Jeanson, 2005), Copyright 2005, with permission from Elsevier. 


\section{Discussion}

The tendency of whether the system aggregates into a single cluster or remains scattered in the environment is a function of $p_{c}$. We observe a change of the qualitative behavior of the steady-state distribution from an unimodal distribution to a bimodal distribution for certain values of $p_{c}$ (Figure 4 ) or the actual communication range in the realistic simulator (Figure 5). At this point, the average number of robots that are searching and that are within a single large cluster are equal. Upon further increasing $p_{c}$ (or the communication range in realistic simulation), the average number of robots in a single large cluster increases at cost of those searching. Indeed, the parameter $p_{c}$ is a function of the robot's speed, and its communication (or team-mate sensing) range (Definition 1). Low values correspond to a low probability to encounter other robots in the arena, either due to reduced mobility or due to limited communication range. It seems that a minimal amount of either mobility or communication range is necessary to achieve aggregation. While we provide experimental evidence based on submicroscopic, realistic simulation only for the influence of communication range but not for the locomotion speed, we conjecture that low communication ranges can be counter-effected with increased mobility, and vice versa.

We also show that the same macroscopic modeling framework that we use for analyzing the robotic simulation can be used to predict the dynamics of a cockroach swarm. This has been possible, as all the behavioral data, i.e., the probabilities to join and leave a cluster as well as the average cockroach speed, and the experimental setup has been made available. Figure 7 suggests, however, that the proposed model can potentially be used to infer missing data and validate hypothesis on parameters, which can not be directly measured such as the effective communication range as in this example. In fact, the effective communication range is the only parameter in (Jeanson et al. 2005) that has not been measured from experimental data, but derived from geometrical considerations based on the assumption that cockroaches communicate via their antennas, which have a limited length.

Although suitable values for $p^{\text {join }}(j)$ and $p^{\text {leave }}(j)$ can be calculated for every desired combination of $N_{j}^{*}$ and $N_{s}^{*}$ by solving a system of linear equations, it is yet unclear whether this equilibrium is stable. Although we are able to provide bounds on the relation between cluster sizes and transition probabilities, further work in the stability analysis of the proposed model is required to create detailed design tools for probabilistic aggregation systems. Nevertheless, the results of our steady state analysis can give already useful guidance in the design process of such self-organized systems. For instance, despite the algorithmic requirements for the proposed algorithm are very well suited for resource-constrained platforms such as the Alice, its communication range does not appear to be sufficient for reliably achieving aggregation into a single aggregate (the communication range of the real Alice robot is limited to $3-4 \mathrm{~cm}$ ) at the chosen speed $\left(4 \frac{\mathrm{cm}}{\mathrm{s}}\right)$. We also note that for a real application the achieved

performance (i.e., reliability of aggregation and time to achieve a single aggregate) might be unsatisfying, but for simpler platforms than the Alice robot, 
e.g., genetically modified cells (Basu et al. 2005) or the I-Swarm robot (size below 3mm) (Valdastri, Corradi, Menciassi, Schmickl, Crailsheim, Seyfried \& Dario 2006), the proposed algorithm might be the only alternative for achieving aggregation.

Better results could be achieved by preventing robots from leaving a cluster and performing collective motions of the aggregates if cluster growth stagnates, which has been proposed in (Poduri \& Sukhatme 2007). This strategy is potentially feasible on the Alice platform, and would allow for coherence of aggregates while preventing deadlocks in sub-optimal solutions (multiple static clusters in the environment).

Another possible improvement is suggested by the motivating cockroach experimental data. Although the proposed model shows close qualitative agreement with the data gathered from experiments with real cockroaches (Jeanson et al. 2005), our model does not explicitly capture an important feature of the real cockroaches. Instead of using a constant probability for leaving a cluster of a certain size as our model does, the cockroaches randomly choose between two values for the probability to leave a cluster. The resulting resting times are drawn from a bimodal distribution with means that are up to two orders of magnitude different. As cockroaches have the same average resting time due to our choice of leaving and joining probabilities and the qualitative behavior of model and experimental data is similar, it is unclear, what the role of the observed bimodal distribution is. The short resting periods strongly affect the coalescence of the system because clusters are becoming more attractive during this short interval, as the temporarily joining cockroach increases the cluster's likelihood to be encountered and its likelihood to be joined. As changing the encountering probability $p_{c}$ can have a similar effect, we conjecture that the cockroaches uses the mechanism of drawing resting times from a bimodal distribution to indeed make aggregation more efficient. Whether this is indeed the case might be answered in the future by carefully estimating $p_{c}$ for the cockroach species and by extending the proposed model to arbitrary, possibly bimodal distributions, for instance using the Gillespie method (Gillespie 1977) for numerical simulation.

We notice that both potential improvements, moving as an aggregate and relying on bimodal leaving-probability distributions, cannot be captured by the current model, which is limited to changes in cluster size by one agent at a time and constant rates. The former limitation is particularly important, as existing clusters could form new ones also in the system proposed in this paper. This is the case when a robot enters the space between two existing clusters. Similarly, our model does not capture cases in which robots leave from the middle of a cluster and leave two clusters of approximately half the size behind. We observed these behaviors to be unlikely in our experimental setup where robots are clustered tightly. Its likelihood increases, however, with increasing communication range of the robots that leads to more sparse deployments. Similarly, specific settings for $p^{\text {join }}(j)$ and $p^{\text {leave }}(j)$ could promote aggregates with minimal connectivity such as lines (Evans, Mermoud \& Martinoli 2010). Such lines could then be broken apart by a robot leaving from the middle. In these cases, the geometry of the resulting clusters is becoming an important aspect of the 
model.

\section{Conclusion}

We adapt a probabilistic model for modeling aggregation phenomena that exhibit not only cluster growth but also cluster dispersion to aggregation of miniature robot swarms. The model links processes that affect the structure of the emerging cluster distribution with the mobility and communication range of individual robots, and is capable of quantitative prediction of self-organized robotic aggregation dynamics. This model has the potential to not only model aggregation in robotic swarms but also self-assembly phenomena on other scales that are be governed by similar construction rules.

We also show that aggregation into a single cluster can be achieved by tuning the behavior of the robots according to behavior observed in gregarious arthropods, but is contigent on a minimal communication range between the robots.

Although the proposed models allow us to qualitatively and quantitatively predict the system dynamics of robot aggregation, and to a limited extent also of cockroach aggregation, its use as a design tool is limited to understanding trade-

offs between communication range and speed as well as bounds on the ratio of aggregation and segregation probabilities. In future work, we are interested in using numerical solutions to the system - whose stability we can assess by inspection - in an optimization framework to find optimal control parameters for the system, which would allow us to generate control parameters that lead to any desired steady-state distribution of aggregates. This is currently not possible as we can assess stability only for special cases of the system.

The model reaches its limitations when the resulting clusters are not tightly packed, but sparse, or form lines. In these cases joining or leaving robots might join multiple clusters or break single clusters apart, which is not captured by our current model. This limits our model to scenarios where aggregates are tightly packed and preferably are disc-shaped. Studying the effect of the various control parameters of the system on the resulting cluster geometry and macroscopic models that allow for the resulting additional state transitions are subject to further work.

\section{Acknowledgements}

We would like to thank Sriram Sankaranarayanan and Dustin Reishus for helpful discussion, as well as helpful feedback from the anonymous reviewers.

\section{References}

Adleman, L., Gopalkrishnan, M., Huang, M., Moisset, P. \& Reishus, D. (2008), On the mathematics of the law of mass-action. Available from arXiv:0810.1108. 
Agassounon, W. \& Martinoli, A. (2002), A Macroscopic Model of an Aggregation Experiment using Embodied Agents in Groups of Time-Varying Sizes, in 'Proc. of the IEEE Conf. on System, Man and Cybernetics SMC-02', pp. $250-255$.

Agassounon, W., Martinoli, A. \& Easton, K. (2004), 'Macroscopic modeling of aggregation experiments using embodied agents in teams of constant and time-varying sizes', Autonomous Robots 17(2-3), 163-191. Special Issue on Swarm Robotics.

Anderson, D. \& Shiu, A. (2010), 'The dynamics of weakly reversible population processes near facets', SIAM Journal on Applied Mathematics 70(6), 18401858.

Basu, S., Gerchman, Y., Collins, C., Arnold, F. \& Weiss, R. (2005), 'A synthetic multicellular system for programmed pattern formation', Nature 434, 1130-1134.

Berg, H. C. (1983), Random Walks in Biology, Princeton University Press.

Camazine, S., Deneubourg, J.-L., Franks, N. R., Sneyd, J., Theraulaz, G. \& Bonabeau, E. (2001), Self-Organization in Biological Systems, Princeton Studies in Complexity, Princeton University Press.

Caprari, G. \& Siegwart, R. (2005), Mobile micro-robots ready to use: Alice, in 'IEEE/RSJ Int. Conf. on Intelligent Robots and Systems (IROS)', Edmonton, Alberta, Canada, pp. 3295-3300.

Correll, N. \& Martinoli, A. (2004), Modeling and optimization of a swarmintelligent inspection system, in 'Proc. of the Int. Symp. on Distributed Autonomous Robotic Systems (DARS)', Springer Distributed Autonomous Robotic Systems (2007), Toulouse, France, pp. 369-378.

Correll, N. \& Martinoli, A. (2006), System identification of self-organized robotic swarms, in 'Proc. of the Int. Symp. on Distributed Autonomous Robotic Systems (DARS)', Springer Distributed Autonomous Robotic Systems, Minneapolis, MN, USA (2006), pp. 31-40.

Correll, N., Rus, D., Bachrach, J. \& Vickery, D. (2009), Ad-hoc wireless network coverage with networked robots that cannot localize, in 'IEEE International Conference on Robotics and Automation', Kobe, Japan, pp. 3878-3885.

Evans, W., Mermoud, G. \& Martinoli, A. (2010), Comparing and modeling distributed control strategies for miniature self-assembling robots, in 'IEEE International Conference on Robotics and Automation', Anchorage, Alaska, pp. 1438-1445.

Feinberg, M. (1995), 'The existence and uniqueness of steady states for a class of chemical reaction networks', Archive for Rational Mechanics and Analysis 132, 311-370. 
Garnier, S., Jost, C., Jeanson, R., Gautrais, J., Grimal, A., Asadpour, M., Caprari, G. \& Theraulaz, G. (2008), 'The embodiment of cockroach aggregation behavior in a group of micro-robots', Artificial Life 14(4), 387-408.

Gillespie, D. (1977), 'Exact stochastic simulation of coupled chemical reactions', The Journal of Physical Chemistry 81(25), 2340-2361.

Halloy, J., Amé, J.-M., Detrain, G. S. C., Caprari, G., Asadpour, M., Correll, N., Martinoli, A., Mondada, F., Siegwart, R. \& Deneubourg, J.-L. (2007), 'Social integration of robots in groups of cockroaches to control self-organized choice', Science 318(5853), 1155-1158.

Jadbabaie, A., Lin, J. \& Morse, A. S. (2003), 'Coordination of groups of mobile autonomous agents using nearest neighbor rules', IEEE Transactions on Automatic Control 48(6), 988-1001.

Jeanson, R., Blanco, S., Fournier, R., Deneubourg, J.-L., Fourcassié, V. \& Theraulaz, G. (2003), 'A model of animal movements in a bounded space', Journal of Theoretical Biology 225, 443-451.

Jeanson, R., Rivault, C., Deneubourg, J.-L., Blanco, S., Fournier, R., Jost, C. \& Theraulaz, G. (2005), 'Self-organized aggregation in cockroaches', Animal Behavior 69, 169-180.

Kazadi, S., Abdul-Khaliq, A. \& Goodman, R. (2002), 'On the convergence of puck clustering systems', Robotics and Autonomous Systems 38(2), 93-117.

Klavins, E. (2007), 'Programmable self-assembly', Control Systems Magazine 24(4), $43-56$.

Lerman, K., Martinoli, A. \& Galystan, A. (2005), A review of probabilistic macroscopic models for swarm robotic systems, in 'Proc. of the SAB 2004 Workshop on Swarm Robotics', Santa Monica, CA, USA, pp. 143-152. Lecture Notes in Computer Science Vol. 3342, Springer-Verlag, Berlin.

Lerman, K. \& Shehory, O. (2000), Coalition formation for large-scale electronic markets, in 'Proc. of the Int. Conf. on Multi-Agent Systems', Boston, MA, p. 0167.

Martinoli, A., Easton, K. \& Agassounon, W. (2004), 'Modeling of swarm robotic systems: A case study in collaborative distributed manipulation', Int. J. of Robotics Research 23(4), 415-436.

Martinoli, A., Ijspeert, A. J. \& Mondada, F. (1999), 'Understanding collective aggregation mechanisms: From probabilistic modelling to experiments with real robots', Robotics 85 Autonomous Systems 29, 51-63. Special Issue on Distributed Autonomous Robotic Systems.

Matthey, L., Berman, S. \& Kumar, V. (2009), Stochastic strategies for a swarm robotic assembly system, in 'Proc. of the IEEE Int. Conf. on Robotics and Automation (ICRA)', Kobe, Japan, pp. 1953-1958. 
Mermoud, G., Brugger, J. \& Martinoli, A. (2009), Towards multi-level modeling of self-assembling micro-systems, in 'Int. Conf. on Autonomous Agents and Multiagent Systems (AAMAS)', Budapest, Hungary, pp. 89-96.

Michel, O. (2004), 'Webots: Professional mobile robot simulation', Journal of Advanced Robotic Systems 1(1), 39-42.

Nembrini, J., Winfield, A. \& Melhuish, C. (2002), Minimalist coherent swarming of wireless connected autonomous mobile robots, in 'Proc. Simulation of Artificial Behaviour SAB 2002', Edinburgh, UK, pp. 273-382.

Parrish, J. K. \& Hammer, W. M. (1997), Animal Groups in Three Dimensions, Cambridge University Press, UK.

Poduri, S. \& Sukhatme, G. (2007), Latency analysis of coalescence in robot groups, in 'IEEE International Conference on Robotics and Automation', Rome, Italy, pp. 3295-3300.

Tanner, H., Jadbabaie, A. \& Pappas, G. (2005), Flocking in teams of nonholonomic agents, Vol. 309 of Lecture Notes in Control and Information Sciences, Springer Verlag, pp. 229-239.

Valdastri, P., Corradi, P., Menciassi, A., Schmickl, T., Crailsheim, K., Seyfried, J. \& Dario, P. (2006), 'Micromanipulation, communication, swarm intelligence issues in a swarm microrobotic platform', Robotics and Autonomous Systems 54, 789-804.

Wang, Y., Liu, J. \& Jin, X. (2003), Modeling agent-based load balancing with time delays, in 'IEEE/WIC International Conference on Intelligent Agent Technology', Australia, pp. 189-195.

Wang, Y. \& Wu, H. (2005), 'Dynamics of a rate equation describing cluster-size evolution', J. Math. Anal. Appl. 310, 379-396.

Whitesides, G. \& Grzybowski, B. (2002), 'Self-assembly at all scales', Science 295, 2418-2421.

Winfield, A., Liu, W., Nembrini, J. \& Martinoli, A. (2008), 'Modelling a wireless connected swarm of mobile robots', Swarm Intelligence 2(2-4), 241-266.

Zangwill, A. (2001), 'Statistical physics: Advances in aggregation', Nature 411, 651-652. 